\title{
Is urine the next source of stem cells?
}

\section{"There is no question that urine offers benefits over dermal fibroblasts and most other tissues in terms of ease of collection and institutional study approval."}

\section{KEYWORDS: induced pluripotent stem cells " neuronal differentiation " reprogramming urine cell culture}

The discovery that pluripotent stem cells can be induced by exposure of somatic cells to reprogramming factors has proven to be one of the most surprising and potentially useful discoveries in regenerative biology [1]. Patient-specific induced pluripotent stem cells (iPSCs) are being widely used to study disease mechanisms and to screen for novel drug therapies for a variety of human disorders.

Fibroblasts were the first somatic cell type to be used in the successful generation of iPSCs and remain in widespread use despite the demonstration that iPSCs can be derived from other readily accessible sources, such as peripheral and cord blood, melanocytes, amniotic and periodontal tissue and hair follicle keratinocytes. The continued popularity of fibroblasts as a source of iPSCs may be historical in the sense that many laboratories began deriving fibroblasts from skin biopsies in anticipation of generating iPSCs and are now in the process of characterizing the disease-specific iPSC lines.

\section{"While we have not yet succeeded in deriving urine induced pluripotent stem cells, we are optimistic that the answer to our question about whether or not urine is the next source of stem cells is 'yes'."}

A practical advantage of dermal fibroblasts over other sources such as peripheral blood is that a trained observer can readily detect nascent iPSC colonies among the adherent fibroblasts in tissue culture. One disadvantage is that skin biopsies are associated with more pain and somewhat higher risk than the collection of blood and, most certainly, of human hair follicles. The discomfort associated with skin biopsies is not a trivial issue. In our institution, for example, the process of human subjects and ethical review is complicated, and lengthened by the oversight committee's knowledge of the availability and the feasibility of using other tissue sources such as hair. This is especially an issue in the case of pediatric disorders where the parent or guardian provides informed consent.

The recent reports that iPSCs can be successfully derived from cells collected from human urine [2-5] could potentially address the aforementioned problems and greatly expand the number of patient-specific stem cells available to the research community. The few thousand epithelial-like cells harvested by centrifugation from a 50-250-ml sample of urine have been used as a source of iPSCs (UiPSCs) and neural progenitors (UiNPCs) [2-5]. UiPSCs, which have been generated using standard reprogramming factors, show the markers of pluripotency that are typical of iPSCs produced from other somatic cell types. UiPSCs can also be differentiated into at least three different lineages: hepatocytes, neurons and cardiomyocytes [5], and disease-specific UiPSCs have been generated for systemic lupus erythematosus [2]. It is also reported that UiNPCs can be reprogrammed directly from the urine epithelial-like cells using episomal vectors that, in contrast to retro- and lenti-viruses, do not integrate into the genome [3]. It is further observed that the UiNPCs have the capacity to self-renew and can be differentiated into different types of functional neurons in vitro, and in vivo upon transplant into newborn rat brain.

There is no question that urine offers benefits over dermal fibroblasts and most other tissues in terms of ease of collection and institutional study approval. The investigations of other groups will confirm whether UiPSCs are similar to standard iPSCs with respect to efficiency of reprogramming as well as degree of pluripotency and capacity for multilineage differentiation. Should this prove to be the case, UiPSCs

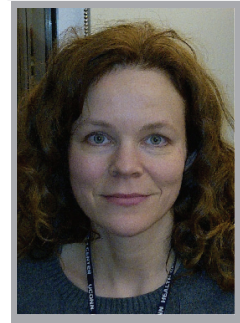

Leann Crandall

Department of Genetics \& Developmental Biology, University of Connecticut, 400 Farmington Avenue, Farmington, CT 06030, USA

and Stem Cell Institute, University of Connecticut, 400 Farmington Avenue, Farmington, CT 06030, USA

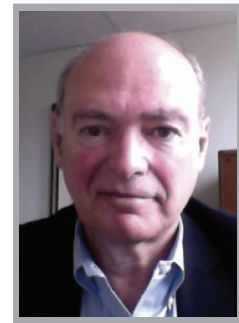

Marc Lalande

Author for correspondence: Department of Genetics \& Developmental Biology, University of Connecticut, 400 Farmington Avenue, Farmington, CT 06030, USA and Stem Cell Institute, University of Connecticut, 400 Farmington Avenue, Farmington, CT 06030, USA Tel.: +18606798349 Fax: +18606798345 lalande@uchc.edu

\section{(6) Regenerative Medicine}


could become a major source of patient-specific stem cells and neural progenitors. As a word of caution, it should be noted that there was great excitement over a report in 2008 that iPSCs could be generated with very high efficiency from hair follicle keratinocytes [6]. Despite the obvious advantages of hair over most, if not all other, human tissues, the stem cell research community has not made extensive use of hair follicles for iPSC derivation. While there may also be unseen technical barriers to the generation of UiPSCs, we can report that cells collected from urine grow vigorously in tissue culture for several weeks. While we have not yet succeeded in deriving UiPSCs, we are optimistic that the answer to our question about whether or not urine is the next source of stem cells is 'yes'.

\section{Financial \& competing interests disclosure}

The authors have no relevant affiliations or financial involvement with any organization or entity with a financial interest in or financial conflict with the subject matter or materials discussed in the manuscript. This includes employment, consultancies, honoraria, stock ownership or options, expert testimony, grants or patents received or pending, or royalties.

No writing assistance was utilized in the production of this manuscript.

\section{References}

1 Yamanaka S. Induced pluripotent stem cells: past, present, and future. Cell Stem Cell 10(6), 678-684 (2012).

2 Chen Y, Luo R, Xu Y et al. Generation of systemic lupus erythematosus-specific induced pluripotent stem cells from urine. Rheumatol. Int. doi:10.1007/s00296-0132704-5 (2013) (Epub ahead of print).
3 Wang L, Huang W, Su H et al. Generation of integration-free neural progenitor cells from cells in human urine. Nat. Methods 10(1), 84-89 (2013).

4 Zhou T, Benda C, Dunzinger $S$ et al. Generation of human induced pluripotent stem cells from urine samples. Nat. Protoc. 7(12), 2080-2089 (2012).
5

Zhou T, Benda C, Duzinger S et al. Generation of induced pluripotent stem cells from urine. J. Am. Soc. Nephrol. 22(7), 1221-1228 (2013).

6 Aasen T, Raya A, Barrero MJ et al. Efficient and rapid generation of induced pluripotent stem cells from human keratinocytes. Nat. Biotechnol. 26(11), 1276-1284 (2008). 